\title{
BMJ Open Efficacy of dexamethasone in reducing the postembolisation syndrome in men undergoing prostatic artery embolisation for benign prostatic hyperplasia: protocol for a single-centre, randomised, double-blind, placebo- controlled trial - the 'DEXAPAE' study
}

\author{
Petra Svarc (D) , ${ }^{1,2}$ Hein Vincent Stroomberg (i) , ${ }^{2,3}$ Ruben Juhl Jensen, ${ }^{1}$ \\ Susanne Frevert, ${ }^{1}$ Mats Håkan Lindh, ${ }^{1}$ Mikkel Taudorf, ${ }^{1,2}$ Klaus Brasso, ${ }^{2,3}$ \\ Lars Lönn, ${ }^{1,2}$ Martin Andreas Røder ${ }^{2,3}$
}

To cite: Svarc P, Stroomberg HV, Juhl Jensen R, et al. Efficacy of dexamethasone in reducing the postembolisation syndrome in men undergoing prostatic artery embolisation for benign prostatic hyperplasia: protocol for a single-centre, randomised, double-blind, placebo-controlled trial-the 'DEXAPAE' study. BMJ Open 2021;11:e047878. doi:10.1136/ bmjopen-2020-047878

- Prepublication history for this paper is available online. To view these files, please visit the journal online (http://dx.doi org/10.1136/bmjopen-2020047878).

Received 10 December 2020 Accepted 08 0ctober 2021

Check for updates

(c) Author(s) (or their employer(s)) 2021. Re-use permitted under CC BY-NC. No commercial re-use. See rights and permissions. Published by BMJ.

For numbered affiliations see end of article.

Correspondence to

Dr Petra Svarc;

petra.svarc@regionh.dk

\section{ABSTRACT}

Introduction Postembolisation syndrome (PES) is the most common side effect of vascular embolisation of solid organs. Although prophylactic corticosteroids are known to reduce the incidence and severity of PES, no trials investigating their efficacy have been conducted in men undergoing prostatic artery embolisation (PAE). We postulate that steroids can have a similar effect in reducing $\mathrm{PES}$ after $\mathrm{PAE}$. This paper describes the rationale and detailed protocol for a randomised controlled trial evaluating the efficacy of dexamethasone (DEXA) in reducing $P E S$ after PAE.

Methods and analysis In this single-centre, randomised, double-blind, placebo-controlled trial, we will enrol 60 individuals undergoing PAE for benign prostatic hyperplasia. Participants will be randomised to receive IV DEXA (24 mg) or placebo (saline). The primary outcomes will be postprocedural fever, pain and quality of life. The secondary outcomes will include postprocedural nausea, postprocedural medicine usage, laboratory parameters (C reactive protein, prostate-specific antigen) and early PAE results.

Ethics and dissemination Ethics approval was obtained from the Danish Committee on Health Research Ethics in the Capital Region (H-20025910). The results from this tria will be disseminated through publication in peer-reviewed journals and national and international presentations. Trial registration number Clinicaltrials.gov identifier: NCT04588857; EudraCT number: 2020-000915-53.

\section{INTRODUCTION}

Benign prostatic hyperplasia $(\mathrm{BPH})$ is a frequent cause of lower urinary tract symptoms (LUTS), with one-fourth of men older than 70 years having moderate to severe LUTS that impair their quality of life (QOL)..$^{1-3}$
Strengths and limitations of this study

- First randomised, double-blind, placebo-controlled trial to examine the efficacy of dexamethasone in reducing symptoms of postembolisation syndrome after prostatic artery embolisation (PAE).

- A wide range of outcomes will be assessed including, but not limited to, postprocedural fever and pain, medicine usage, prostate-specific antigen values and early PAE outcomes.

- The study is powered to show a clinically important difference in the primary outcome measurements.

- Research staff and statistician analysing the data will be blinded to randomisation group.

- Potential limitation: good patient compliance is paramount for study success, as most outcome measures will be self-reported by the patients.

Prostatic artery embolisation (PAE) is a new minimally invasive technique proven effective in reducing LUTS comparable to mainstay treatment-the transurethral resection of the prostate. $^{4-7}$

The most common side effect of vascular embolisation of solid organs is a collection of inflammation-related symptoms known as the postembolisation syndrome (PES) ${ }^{8}$ The aetiology of PES is not fully explored; however, it is hypothesised that tissue hypoxia and cell death lead to the release of tissue breakdown products, inflammatory mediators and vasoactive substances. ${ }^{8} 9$ The syndrome is characterised by influenza-like symptoms, pain, nausea, fever, and in the case of PAE, dysuria and transient worsening of LUTS. ${ }^{8}$ 
Leucopenia, leucocytosis, elevation of $\mathrm{C}$ reactive protein (CRP) and other raised inflammatory parameters can be observed. ${ }^{9}$ There is currently no consensus on both the definition and classification of PES and its incidence varies widely in the literature, from $0 \%$ to $100 \%{ }^{6}{ }^{10-13}$ Reported incidence in other anatomical sites varies from $40 \%$ in uterine artery embolisation to $89 \%$ in renal angiomyolipoma embolisation. ${ }^{9} 14$ Empirical observations from our own group of men suggest that PES occurs in up to $90 \%$ with a varying degree of severity, ranging from admission to hospital to only mild discomfort 2-3 days after intervention.

PES is a self-limiting condition that is treated symptomatically with a combination of analgesics, antiemetics and antipyretics. However, PES can be so severe that the patients experience high fever, shivers, dysuria and urgency, mimicking septicaemia from the urinary tract. As shown by Ganguli et al in uterine artery embolisation, leucocytosis is frequent after solid organ embolisation further complicating the discerption of PES from infection. ${ }^{9}$ It is a clinical challenge to avoid needless antibiotics treatment in those situations. A subset of patients may need hospital admission for observation, especially those with fever. Furthermore, most patients experience PES as very uncomfortable and have a temporary decrease in QOL. Usually, PES resolves within 5 days after PAE.

Following the inflammation hypothesis, prophylactic corticosteroids were used and proven successful in reducing the incidence, severity and duration of PES after renal angiomyolipoma ablation, endovascular abdominal aortic repair and transcatheter arterial chemoembolisation of the liver. ${ }^{14-16}$ The last two studies were conducted as double-blind randomised placebocontrolled trials with a low risk of bias, providing good evidence quality for corticosteroid usage. Administration of a single-dose perioperative corticosteroid was not associated with any significant side effects in a meta-analysis of RCTs done by DeOliveira et $a l .{ }^{17}$ No similar studies were conducted concerning PES after PAE, and symptomatic therapy is still the mainstay treatment.

In this study, we will evaluate the efficacy of single highdose postprocedural dexamethasone (DEXA) administration in reducing PES after PAE. PES has several manifestations and varies greatly between patients. As there is currently no international consensus on an exact definition of PES, choosing a suitable primary outcome parameter for this study is challenging. To our experience, fever and severe pelvic floor pain are the symptoms most frequently causing patient concern, increased medicine usage and contact to our emergency out patient clinic. In a small pilot study that the sample size calculation is based on, almost all patients experienced fever following PAE. Thus, the main objective of this study is to demonstrate a decrease in postprocedural body temperature and pain, as well as an increase in QOL, with DEXA compared with placebo. Additionally, we aim to demonstrate that DEXA compared with placebo decreases postprocedural medication usage, rate of hospital admissions, inflammatory parameters and short-term post-PAE LUTS worsening. Finally, early PAE outcomes for both groups will be collected.

\section{METHODS AND ANALYSIS}

\section{Study design and setting}

This study is designed as a single-centre, double-blind, randomised, placebo-controlled clinical trial comparing single postprocedural $24 \mathrm{mg}$ IV dose of DEXA with placebo (isotonic solution of sodium chloride). The choice of $24 \mathrm{mg}$ DEXA is based on the results of several studies and meta-analyses exploring the anti-inflammatory effects of steroids given perioperatively at various doses. ${ }^{17-21}$ Additionally, DEXA is chosen over other steroids due to its long biological action and favourable side-effect profile.

The study will be conducted at Rigshospitalet, Copenhagen, Denmark, starting in January 2021 with an estimated completion date in the first half of 2022. Rigshospitalet is currently one of only two centres in Denmark offering PAE, with up to 120 cases performed annually, which ensures that the number of patients needed for this study will be recruited within the allocated time frame.

\section{Study population}

Participants will be recruited among patients with LUTS due to BPH currently followed at the Department of Urology, Rigshospitalet, during one of their regular visits. Treatment-responsible physician, a urology consultant, will assess whether the patient is eligible for participation in the study. The study flow and eligibility criteria are seen in figure 1. Inclusion criteria for the study reflect the current PAE indications at our institution. On the other hand, exclusion criteria consist of current urological contraindications to PAE, contraindications for catheterbased interventions and contraindications for high-dose DEXA administration. The study will not be advertised in any way. All study visits will take place at the Department of Urology, Rigshospitalet. PAE will be performed at the Department of Radiology, Rigshospitalet.

\section{Outcome assessment}

All outcome measures during the first month following PAE, with the exception of blood tests, will be selfreported by the participants in a diary they will receive on the day of the intervention. Outcome measures 3 and 6 months following PAE will be assessed during a follow-up visit to the urologist (as part of the standard postprocedural protocol for all patients), where the diary will also be collected. Study physician will contact the participants 5 days and 1 month after the intervention, to assess compliance and answer any eventual questions. CRP will be measured at baseline and 2 days following PAE in an outpatient laboratory. Likewise, prostate-specific antigen (PSA) will be followed at baseline, 2 days, 1 month, 3 months and 6 months. All outcome measures collected 


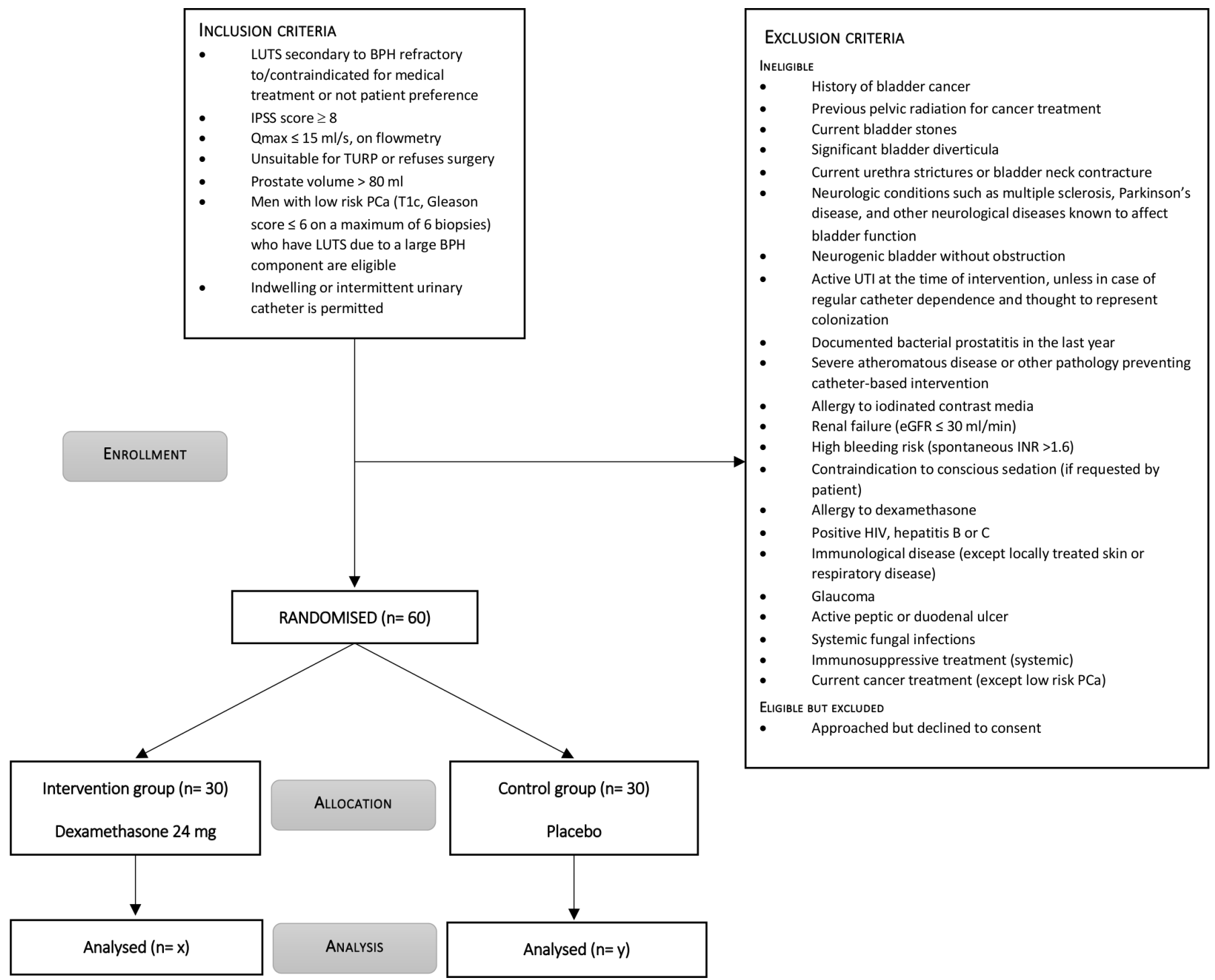

Figure 1 Study flow and eligibility criteria. BPH, benign prostatic hyperplasia; eGFR, estimated glomerular filtration rate; INR, international normalised ratio; IPSS, International Prostate Symptom Score; LUTS, lower urinary tract symptoms; PCa, prostate cancer; Qmax, maximum flow velocity; TURP, transurethral resection of the prostate; UTI, urinary tract infection.

during the trial at various time points are summarised in table 1 .

\section{Primary outcome}

The primary outcome for the DEXAPAE study is to determine the efficacy of $24 \mathrm{mg}$ DEXA, compared with placebo control, in reducing the symptoms of PES after PAE, as indicated by the following: mean rectal body temperature in degrees Celsius 2 days after PAE compared with baseline, mean postprocedural pain and mean QOL for the first 5 days post-PAE, the latter two measured as pain severity and pain interference scores on Brief Pain Inventory-Short Form (BPI-SF) questionnaire, respectively. ${ }^{22}$

\section{Secondary outcomes}

The effect of DEXA versus placebo will be assessed for the following secondary outcomes: postprocedural inflammatory response markers (CRP and PSA), need for postprocedural medication (analgesics, antipyretics, antiemetics), incidence of hospital admission, LUTS severity measured on International Prostate Symptom Score (IPSS) questionnaire, erectile function measured on International Index of Erectile Function (IIEF-5) questionnaire, prostate volume on transrectal ultrasound, uroflowmetry, residual urine, incidence of urinary tract infections, incidence of acute urinary retention and incidence of side effects of PAE. Laboratory values, IPSS and IIEF-5 scores, prostate volume and urodynamic measurements (uroflowmetry and residual urine) will be assessed as a change from baseline to all outcome measurement time points.

\section{Screening}

Treatment-responsible physician will assess, based on the inclusion and exclusion criteria, if the patient is eligible for the study. If the patient is qualified and gives verbal informed consent, the trial's advantages, side effects and 
Table 1 Outcome measures at each time point

\section{Screening Day 0 (PAE) Day 1 Day 2 Day 3 Day 4 Day 51 month 3 months 6 months}

\begin{tabular}{|c|c|c|c|c|c|c|c|c|c|c|}
\hline BT & & $x$ & $x$ & $x$ & $x$ & $x$ & $x$ & & & \\
\hline BPI-SF & & $x$ & $x$ & $x$ & $x$ & $x$ & $x$ & & & \\
\hline Medication usage & & $x$ & $x$ & $\mathrm{x}$ & $x$ & $x$ & $x$ & & & \\
\hline Dysuria & & $x$ & $x$ & $x$ & $x$ & $x$ & $x$ & & & \\
\hline Blood glucose * & & $x$ & $x$ & $x$ & $x$ & $x$ & $x$ & & & \\
\hline IIEF-5 & $x$ & & & & & & & $x$ & $x$ & $x$ \\
\hline PSA & $x$ & & & $x$ & & & & $x$ & $x$ & $x$ \\
\hline CRP & $x$ & & & $x$ & & & & & & \\
\hline Uroflowmetry & $x$ & & & & & & & & $x$ & $x$ \\
\hline TRUS & $x$ & & & & & & & & $x$ & $x$ \\
\hline Acute urinary retention & & $x$ & $x$ & $x$ & $x$ & $x$ & $x$ & & & \\
\hline
\end{tabular}

*Blood glucose is only collected for participants with diabetes.

BPI-SF, Brief Pain Inventory-Short Form; BT, body temperature; CRP, C reactive protein; IIEF-5, International Index of Erectile Function-5; IPSS, International Prostate Symptom Score;PAE, prostatic artery embolisation; PSA, prostate-specific antigen; TRUS, transrectal ultrasound; UTI, urinary tract infection.

disadvantages will be discussed in detail, and the patient will be given a patient summary of the protocol, patient information and other relevant study material. The patient is offered a minimum of 24 hours of decision time and will be scheduled for a follow-up visit, where eventual questions will be answered, and informed consent will be collected. The patient is offered to bring a relevant attendant (spouse, family, friend) to the follow-up visit. If the patient wishes a prolonged decision period, a new appointment will be arranged. After the elapsed period of reflection, the patient will sign a written informed consent together with the trial physician. The patient can withdraw the informed contest at any time without explanation.

\section{Sample size}

A pilot study conducted at the Department of Urology, Rigshospitalet on four patients undergoing PAE (without DEXA administration) showed a mean postprocedural rectal temperature of $37.8^{\circ} \mathrm{C}$ (SD 0.38) 2 days post-PAE. Day 2 is chosen as study target as it had the highest mean temperature of the 5 days in which the temperature was measured. The goal reduction is from mean temperature on day 2 (subfebrile) to a mean of $37.4^{\circ} \mathrm{C}$ (afebrile). A one-sided two-sample t-test power calculation with $80 \%$ power and a significance of 0.05 gives a required sample size of 28 patients in each group or 56 patients in total.

In the literature, a 3-point decrease (SD 2) on the BPI-SF questionnaire is considered clinically relevant. ${ }^{23} 24$ With that in mind, a one-sided two-sample t-test power calculation with $80 \%$ power and a significance of 0.05 gives a required sample size of 7 patients in each group or 14 patients in total.

In conclusion, this study will include 30 patients in each group (60 in total) to compensate for eventual dropouts. Dropouts are defined as patients who, after inclusion, either do not receive the allocated trial medication or are lost to follow-up for the primary outcomes.

The study has not been powered for the secondary outcome measures.

\section{Randomisation and allocation concealment}

Participants will be randomised on the day of the intervention. Dropouts will be replaced by the same randomisation number to ensure equal distribution. Allocation of randomisation numbers for the dropouts will be blinded.

Randomisation will be performed as permuted block randomisation with a block size of 6 and 1:1 allocation ratio using a secure online randomisation system to ensure allocation concealment.

Participants, care providers (ie, interventional radiologists performing the procedure, urologists responsible for patient selection and follow-up, research nurses involved in the trial) and researchers involved in data collection and analysis will be blinded to allocation. A physician not otherwise involved in the study will prepare the medication in a private room prior to PAE to ensure blinding is preserved. No masking of syringe content is necessary as both DEXA and saline are clear, colourless liquids. A nurse blinded to the syringe content will administer the medication immediately following PAE. 
The treatment allocation will only be unblinded in case of a suspected serious adverse reaction to the study medication, the management of which might be influenced by knowledge of the allocation.

The unmasked randomisation list will be kept by a physician not involved in the study in a locked drawer and will not be given to the principal investigator until all trial data are collected.

\section{Statistical analysis}

All analyses will be conducted by blinded research staff and supervised by a statistician. The normality of distribution will be assessed with a q-q plot and the Shapiro-Wilk test. Parametric data will be analysed using the independent Student's t-test, and non-parametric data will be analysed using the Mann-Whitney U test. Categorical variables will be evaluated using the chi-squared test or Fisher's exact test, when appropriate. All values will be expressed as mean $\pm \mathrm{SD}$, median (range) or the number of patients (proportion). $\mathrm{p}<0.05$ will be considered to be statistically significant.

The nature of missing data will be examined to consider appropriate approaches. For the primary outcomes, data from all the participants for whom there is at least one temperature measurement on day 2 post-PAE and at least four completed BPI-SF questionnaires during the first 5 days post-PAE will be included in the statistical analysis. The area under the curve (AUC) can be calculated from available temperature measurements and BPI-SF scores even if some are missing, by using linear interpolation.

Statistical analysis will be undertaken in RStudio using R V.3.2 or later if available. ${ }^{25}$

\section{Data management}

Study data will be collected and managed using Research Electronic Data Capture (REDCap) electronic data capture tools hosted at Rigshospitalet, Denmark. ${ }^{26}{ }^{27}$ REDCap is a secure, web-based software platform designed to support data capture for research studies, providing an intuitive interface for validated data capture, audit trails for tracking data manipulation and export procedures, automated export procedures for seamless data downloads to common statistical packages and procedures for data integration and interoperability with external sources. The database will not be unblinded until data collection has been declared as complete. The study team will be responsible for all data entry and quality control. All data will be archived in REDCap for 5 years after the study is ended, in accordance with relevant local and European Union (EU) laws.

\section{Safety monitoring}

At both follow-up visits, participants will be asked to report any adverse events (AEs) that might have occurred since their previous visit. AEs will also be identified and collected from patient journals. All AEs judged by either the reporting investigator or the sponsor as having a reasonable causal relationship to the study drug qualify as adverse reactions. All serious AEs (SAEs) will be reported to the sponsor by the investigator no later than 24 hours after their occurrence during the whole length of the study. This immediate report will be supplemented by a follow-up report that allows the sponsor to determine whether the SAE requires a reassessment of the risk-benefit ratio of the study. The investigator will notify the sponsor immediately if a suspected unsuspected serious adverse reaction (SUSAR) occurs, to allow facilitation of unblinding if necessary. The sponsor or the investigator will notify the relevant Ethics Committee and the Danish Medicines Agency within 24 hours if during the clinical trial a SUSAR occurs. Once a year throughout the study period, the sponsor or the investigator will submit a list of all suspected serious adverse reactions (expected and unexpected) that occurred in the period to the Ethics Committee and the Danish Medicines Agency. The report will include an assessment of the safety of the study participant.

The study will be independently monitored by the Good Clinical Practice (GCP) Unit at the University of Copenhagen. They will periodically assess, following local guidelines, if the study is conducted according to the protocol and note any eventual protocol deviations that may influence patient safety or study outcomes.

\section{Patient and public involvement}

Neither the patients nor the public were involved in the planning or design of the study.

\section{Ethics and dissemination}

This study has been approved by the Danish Committee on Health Research Ethics in the Capital Region (Id: H-20025910) on 1 October 2020. The study will be conducted in accordance with the protocol, ICH-GCP guidelines, the Helsinki Declaration and the applicable local guidelines and laws. The established procedures for quality control will be followed. All participants will provide written informed consent prior to involvement in the study. Participants may withdraw their consent at any time without explanation. A plain Danish summary of the study results will be sent to the study participants once data analysis has been completed.

The results will be published in a peer-reviewed journal and presented at regional, national and international scientific meetings as appropriate.

\section{Author affiliations}

${ }^{1}$ Department of Radiology, Rigshospitalet, Copenhagen, Denmark

${ }^{2}$ Faculty of Health and Medical Sciences, University of Copenhagen, Copenhagen, Denmark

${ }^{3}$ Copenhagen Prostate Cancer Center (CPC), Department of Urology, Rigshospitalet, Copenhagen, Denmark

\section{Twitter Petra Svarc @petrasvarc}

Contributors LL, MAR and PS conceived the study and initiated the study design. $\mathrm{LL}$ is the study sponsor. MAR is the principal investigator. HVS is responsible for statistical support, analysis and the sample size calculations provided. PS wrote the first manuscript draft. LL, PS, RJJ, SF, MHL and MT are responsible for trial implementation and trial-specific procedures at the Department of Radiology. MAR, HVS and KB are responsible for trial implementation and trial-specific procedures at the Department of Urology. All authors have reviewed and commented on this manuscript.

Funding The authors have not declared a specific grant for this research from any funding agency in the public, commercial or not-for-profit sectors. 
Competing interests None declared.

Patient and public involvement Patients and/or the public were not involved in the design, or conduct, or reporting, or dissemination plans of this research.

Patient consent for publication Not applicable.

Provenance and peer review Not commissioned; externally peer reviewed.

Open access This is an open access article distributed in accordance with the Creative Commons Attribution Non Commercial (CC BY-NC 4.0) license, which permits others to distribute, remix, adapt, build upon this work non-commercially, and license their derivative works on different terms, provided the original work is properly cited, appropriate credit is given, any changes made indicated, and the use is non-commercial. See: http://creativecommons.org/licenses/by-nc/4.0/.

\section{ORCID iDs}

Petra Svarc http://orcid.org/0000-0003-0753-3806

Hein Vincent Stroomberg http://orcid.org/0000-0002-7293-9506

\section{REFERENCES}

1 Roehrborn CG. Benign prostatic hyperplasia: an overview. Rev Urol 2005;7(Suppl 9):S3-14.

2 Thorpe A, Neal D. Benign prostatic hyperplasia. The Lancet 2003:361:1359-67.

3 Irwin DE, Kopp ZS, Agatep B, et al. Worldwide prevalence estimates of lower urinary tract symptoms, overactive bladder, urinary incontinence and bladder outlet obstruction. BJU Int 2011;108:1132-8.

4 Malling B, Røder MA, Brasso K, et al. Prostate artery embolisation for benign prostatic hyperplasia: a systematic review and meta-analysis. Eur Radiol 2019;29:287-98.

5 Gao Y-an, Huang Y, Zhang R, et al. Benign prostatic hyperplasia: prostatic arterial embolization versus transurethral resection of the prostate--a prospective, randomized, and controlled clinical trial. Radiology 2014;270:920-8.

6 Carnevale FC, Iscaife A, Yoshinaga EM, et al. Transurethral resection of the prostate (TURP) versus original and Perfected prostate artery embolization (PAE) due to benign prostatic hyperplasia (BPH): preliminary results of a single center, prospective, Urodynamic-Controlled analysis. Cardiovasc Intervent Radiol 2016;39:44-52

7 Abt D, Hechelhammer L, Müllhaupt G, et al. Comparison of prostatic artery embolisation (PAE) versus transurethral resection of the prostate (TURP) for benign prostatic hyperplasia: randomised, open label, non-inferiority trial. BMJ 2018;361:k2338.

8 Moreira AM, de Assis AM, Carnevale FC, et al. A review of adverse events related to prostatic artery embolization for treatment of bladder outlet obstruction due to BPH. Cardiovasc Intervent Radiol 2017;40:1490-500.

9 Ganguli S, Faintuch S, Salazar GM, et al. Postembolization syndrome: changes in white blood cell counts immediately after uterine artery embolization. J Vasc Interv Radiol 2008;19:443-5.
10 Svarc P, Taudorf M, Nielsen MB, et al. Postembolization syndrome after prostatic artery embolization: a systematic review. Diagnostics 2020;10:659.

11 Kurbatov D, Russo GI, Lepetukhin A, et al. Prostatic artery embolization for prostate volume greater than $80 \mathrm{~cm} 3$ : results from a single-center prospective study. Urology 2014;84:400-4.

$12 \mathrm{Yu}$ SCH, Cho CCM, Hung EHY, et al. Prostate artery embolization for complete urinary outflow obstruction due to benign prostatic hypertrophy. Cardiovasc Intervent Radiol 2017;40:33-40.

13 Amouyal G, Thiounn N, Pellerin O, et al. Clinical results after prostatic artery embolization using the Perfected technique: a single-center study. Cardiovasc Intervent Radiol 2016;39:367-75.

14 Bissler JJ, Racadio J, Donnelly LF, et al. Reduction of postembolization syndrome after ablation of renal angiomyolipoma Am J Kidney Dis 2002;39:966-71.

15 de la Motte L, Kehlet H, Vogt K, et al. Preoperative methylprednisolone enhances recovery after endovascular aortic repair: a randomized, double-blind, placebo-controlled clinical trial. Ann Surg 2014;260:540-9.

16 Ogasawara S, Chiba T, Ooka Y, et al. A randomized placebocontrolled trial of prophylactic dexamethasone for transcatheter arterial chemoembolization. Hepatology 2018;67:575-85.

17 De Oliveira GS, Almeida MD, Benzon HT, et al. Perioperative single dose systemic dexamethasone for postoperative pain: a meta-analysis of randomized controlled trials. Anesthesiology 2011;115:575-88.

18 Holte K, Kehlet H. Perioperative single-dose glucocorticoid administration: pathophysiologic effects and clinical implications. $J$ Am Coll Surg 2002;195:694-712.

19 Tønnesen E, Wanscher M, Höhndorf K, et al. Effect of methylprednisolone on the cytokine response in patients undergoing lung surgery. Acta Anaesthesiol Scand 1993;37:410-4.

20 Lunn TH, Kehlet H. Perioperative glucocorticoids in hip and knee surgery - benefit vs. harm? A review of randomized clinical trials. Acta Anaesthesiol Scand 2013;57:823-34.

21 Kristeller JL, Jankowski A, Reinaker T. Role of corticosteroids during cardiopulmonary bypass. Hosp Pharm 2014;49:232-6.

22 Cleeland CS, Ryan KM. Pain assessment: global use of the brief pain inventory. Ann Acad Med Singap 1994;23:129-38.

23 Marcus J, Lasch K, Wan Y, et al. An assessment of clinically important differences on the worst pain severity item of the modified brief pain inventory in patients with diabetic peripheral neuropathic pain. Pain Res Manag 2018;2018:1-8.

24 Hanna M, Thipphawong J, 118 Study Group. A randomized, doubleblind comparison of OROS(R) hydromorphone and controlled-release morphine for the control of chronic cancer pain. BMC Palliat Care 2008;7:17.

25 RStudio Team. RStudio: Integrated Development for R. Boston, MA: PBC, 2020. Available: http://www.rstudio.com/ [Accessed 1 Oct 2020].

26 Harris PA, Taylor R, Thielke R, et al. Research electronic data capture (REDCap)--a metadata-driven methodology and workflow process for providing translational research informatics support. J Biomed Inform 2009;42:377-81.

27 Harris PA, Taylor R, Minor BL, et al. The REDCap consortium: building an international community of software platform partners. $J$ Biomed Inform 2019;95:103208. 